PROCEEDINGS OF THE AMERICAN MATHEMATICAL SOCIETY

Volume 127, Number 4, April 1999, Pages 1183-1190

S 0002-9939(99)04719-X

\title{
NONUNIQUENESS OF PHASE TRANSITIONS NEAR THE MAXWELL LINE
}

\author{
S. BENZONI-GAVAGE
}

(Communicated by Jeffrey Rauch)

\begin{abstract}
We consider the description of propagating phase boundaries in a van der Waals fluid by means of viscocapillary profiles, which are viewed as heteroclinic orbits connecting nonhyperbolic fixed points of a five dimensional dynamical system. A bifurcation analysis enables us to show that, for small viscosities, some distinct propagating phase boundaries share the same metastable state on one side of the front.
\end{abstract}

\section{INTRODUCTION}

We are concerned with propagating phase boundaries in an isothermal van der Waals fluid. The underlying discontinuities have fewer degrees of freedom than the classical ones for compressible fluids - shocks or contact discontinuities. As a matter of fact, phase transitions are required to have viscocapillary profiles [6], which implies, as for undercompressive shocks [3], an additional condition to the Rankine-Hugoniot conditions. While shock waves or contact discontinuities need the fluid's state on one side of the front and the front speed to be determined, we expect that only one of these data determines phase transitions. Therefore, phase transitions are commonly parametrized by one of the fluid's states. This state may be any of them in the case of an inviscid fluid, in which phase transitions are reversible [1]. However, the purpose of this paper is to show that dissipative phase transitions are characterized by the state that receives the mass transfer - which is in accordance with the uniqueness theorems stated in [2] or [7]. This state is in a physically speaking stable phase, i.e., it lies outside the Maxwell points, which correspond to the only phase transition with no mass transfer. On the other hand, the metastable states - lying between the Maxwell points - that are close enough to one of these points define at least two phase transitions. This nonuniqueness result, unlike Grinfeld's one for instance [4], concerns profiles that remain close to the Maxwell profile.

\section{THE ZERO VISCOSITY BIFURCATION}

1.1. Viscocapillary profiles. Let us consider a van der Waals type smooth pressure law $p: v \in] b,+\infty[\mapsto p(v)>0$ that is decreasing except on some interval

Received by the editors July 16, 1997.

1991 Mathematics Subject Classification. Primary 76T05, 35M10, 34C37; Secondary 35L67, $58 \mathrm{~F} 14$.

Key words and phrases. Phase transitions, profiles, heteroclinic orbits.

(C)1999 American Mathematical Society 
] $v_{\star}, v^{\star}$ [, where it is increasing, and such that $\lim _{v \rightarrow b} p(v)=+\infty, \lim _{v \rightarrow+\infty} p(v)=$ 0 . Then there exists a unique $\left.\left(v_{m}, v_{M}, \bar{p}\right) \in\right] b, v_{\star}[\times] v^{\star},+\infty\left[\times \mathbb{R}^{+*}\right.$, called the Maxwell equilibrium, such that the equal area rule:

$$
\int_{v_{m}}^{v_{M}}(\bar{p}-p(v)) d v=0, \bar{p}=p\left(v_{m}\right)=p\left(v_{M}\right),
$$

holds. We shall base our analysis on these points.

We assume the motion of the fluid is governed by the Euler equations supplemented with a viscosity coefficient $\mu \geq 0$ and a capillarity coefficient $\varepsilon>0$ :

$$
\rho_{t}+(\rho u)_{x}=0,(\rho u)_{t}+\left(\rho u^{2}+p\right)_{x}=\mu u_{x x}-\varepsilon v_{x x x},
$$

where $\rho:=1 / v$ denotes the density, $p=p(v)$ the pressure and $u$ the velocity. According to the Slemrod criterion [6], a phase transition of speed $s$ between the specific volumes $\left.\left.v_{l} \in\right] b, v_{\star}\right]$ and $v_{r} \in\left[v^{\star},+\infty[\right.$ is admissible if it admits a viscocapillary profile, that is to say a travelling wave of the form $(x, t) \in \mathbb{R} \times \mathbb{R}^{+} \mapsto$ $(v((x-s t) / \sqrt{\varepsilon}), u((x-s t) / \sqrt{\varepsilon}))$ solution to $(1.2)$ such that $v(-\infty)=v_{l}$ and $v(+\infty)=v_{r}$. If such a profile exists, then the function $\xi \mapsto u(\xi)$ has some limits $u_{l}$ and $u_{r}$ at $-\infty$ and $+\infty$ respectively and we have

$$
\begin{aligned}
\rho_{l}\left(u_{l}-s\right) & =\rho_{r}\left(u_{r}-s\right), \\
\rho_{l} u_{l}\left(u_{l}-s\right)+p\left(v_{l}\right) & =\rho_{r} u_{r}\left(u_{r}-s\right)+p\left(v_{r}\right) .
\end{aligned}
$$

Equations (1.3) are nothing but the Rankine-Hugoniot conditions of the Euler equations. They can also be written by means of the mass transfer $j:=\rho(u-s)$ as:

$$
u_{l}-j v_{l}=u_{r}-j v_{r}, p\left(v_{l}\right)+j^{2} v_{l}=p\left(v_{r}\right)+j^{2} v_{r} .
$$

Owing to (1.4) and an elementary integration, we can eliminate the velocity $u$ from the question of existence of viscocapillary profiles. Eventually, a phase transition of mass transfer $j$ between $v_{l}$ and $v_{r}$ is admissible if there exists a smooth solution of the ordinary differential equation

$$
v^{\prime \prime}=\nu j v^{\prime}+p\left(v_{l}\right)+j^{2} v_{l}-p(v)-j^{2} v,
$$

where $\nu:=\mu / \sqrt{\varepsilon}$, such that

$$
v(-\infty)=v_{l} \text { and } v(+\infty)=v_{r} .
$$

We have preferred here an Eulerian formulation of the motion since it allows us to consider (planar) multidimensional phase transitions. However, a Lagrangian formulation would lead to the same differential equation as (1.5) except that $-j$ would be replaced by the (relative) speed of the boundary. So this choice does not play any role in the analysis.

In the particular case $j=0$, the profile equation (1.5) gets much simpler. Hence, whatever the viscosity of the fluid, the only admissible phase transition $\left.\left(v_{l}, v_{r}\right) \in\right] b, v_{\star}[\times] v^{\star},+\infty[$ with no mass transfer corresponds to the Maxwell points $v_{l}=v_{m}$ and $v_{r}=v_{M}$ defined by (1.1). On the other hand, the existence of strictly capillary profiles - i.e. when $\mu=0$ - is linked to a generalized equal area rule (see Figure 1), that is to say:

$$
\int_{v_{l}}^{v_{r}}\left(p_{l}-p(v)+j^{2}\left(v_{l}-v\right)\right) d v=0, \frac{p\left(v_{r}\right)-p\left(v_{l}\right)}{v_{r}-v_{l}}=-j^{2} .
$$


This shows that for an inviscid fluid, admissible phase transitions $\left(v_{l}, v_{r}\right) \in$ ]$b, v_{\star}[\times] v^{\star},+\infty[$ are such that

$$
v_{l} \leq v_{m} \text { and } v_{r} \geq v_{M} .
$$

If $\mu>0$, then phase transitions behave differently. We know (see [5], [7]) that for $j$ near 0 , the admissible phase transitions read $\left(v_{l}=v_{l}(j), v_{r}=v_{r}(j)\right)$ with $v_{l}(0)=v_{m}, v_{r}(0)=v_{M}$ and $d_{j} v_{l, r}(0) \neq 0$. If we differentiate the second equation of (1.4), we see from $d_{v} p\left(v_{m}\right)<0$ and $d_{v} p\left(v_{M}\right)<0$ that $d_{j} v_{l}(0)$ and $d_{j} v_{r}(0)$ are strictly of the same sign. This is not compatible with (1.7) and one of the states lies in the so-called metastable region $] v_{m}, v_{\star}[\cup] v^{\star}, v_{M}[$. (This was already pointed out in [8].) So, there is a bifurcation at $\mu=0$ that we have to analyze.

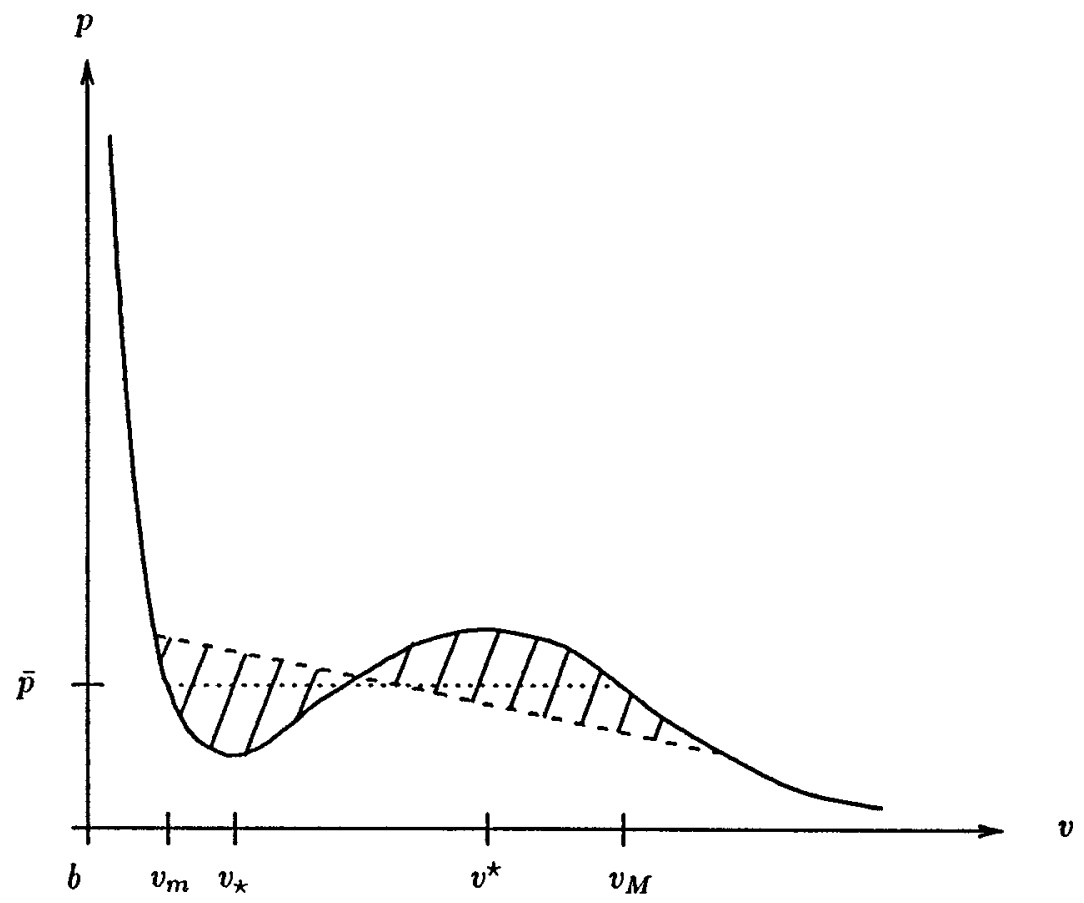

Figure 1. The generalized equal area rule

1.2. The augmented dynamical system. Equation (1.5) may be rewritten as a first order system in the phase plane. When $j=0$, this system has a heteroclinic orbit connecting the saddle points $\left(v_{m}, 0\right)$ and $\left(v_{M}, 0\right)$ that is parametrized by $\xi \mapsto\left(V(\xi), V^{\prime}(\xi)\right)$, where $V$ is called the Maxwell profile and is the unique solution up to a translation to

$$
V^{\prime \prime}=\bar{p}-p(V), \quad V(-\infty)=v_{m} \text { and } V(+\infty)=v_{M}
$$

We shall concentrate on the profiles that are close to the Maxwell profile, when $(j, \nu)$ is close to $(0,0)$. Therefore, we add three trivial equations to the original system and we consider the system of five equations:

$$
v^{\prime}=w, \quad w^{\prime}=\nu j w+\pi-p(v)-j^{2} v, \pi^{\prime}=0, \quad j^{\prime}=0, \quad \nu^{\prime}=0,
$$

one obvious solution of which is $\bar{U}: \xi \mapsto\left(V(\xi), V^{\prime}(\xi), \bar{p}, 0,0\right)$. 
Lemma 1.1. There exist two surfaces $\Sigma_{m, M} \subset \mathbb{R}^{5}$ containing

$$
U_{m, M}:=\left(v_{m, M}, 0, \bar{p}, 0,0\right)
$$

respectively, locally parametrized by $(j, \nu)$ and such that, for all $U_{l} \in \Sigma_{m}$, there is a global solution $\xi \mapsto U(\xi)$ to system (1.9) satisfying $U(-\infty)=U_{l}$ and $U(+\infty)=$ $U_{r} \in \Sigma_{M}$.

Proof. It follows the ideas of the similar result for a fixed viscosity [5], [7]. We shall denote by $\Gamma$ the heteroclinic orbit connecting $U_{m}$ to $U_{M}$ in $\mathbb{R}^{5}$ that is described by the global solution $\bar{U}$. The matrices of the linearized system of (1.9) at points $U_{m, M}$ read

$$
A_{m, M}=\left(\begin{array}{ccccc}
0 & 1 & 0 & 0 & 0 \\
C_{m, M}^{2} & 0 & 1 & 0 & 0 \\
0 & 0 & 0 & 0 & 0 \\
0 & 0 & 0 & 0 & 0 \\
0 & 0 & 0 & 0 & 0
\end{array}\right),
$$

where $C_{m, M}^{2}:=-d_{v} p\left(v_{m, M}\right)$. They have 0 as an eigenvalue of multiplicity 3 . Consequently, system (1.9) has dimension 3 center manifolds $\mathcal{W}_{m, M}^{c}$ around the fixed points $U_{m, M}$ that are locally equal to the dimension 3 manifolds of the fixed points $\left(v, 0, p(v)+j^{2} v, j, \nu\right)$ close to $U_{m, M}$. Their tangent spaces $T_{U_{m, M}} \mathcal{W}_{m, M}^{c}$ are generated by

$$
\left(1,0,-C_{m, M}^{2}, 0,0\right),(0,0,0,1,0) \text { and }(0,0,0,0,1) .
$$

Moreover, system (1.9) has an unstable curve $\mathcal{W}_{m}^{u}$ at point $U_{m}$ that is tangent to the eigenvector $\left(1, C_{m}, 0,0,0\right)$ of $A_{m}$, as well as a stable curve $\mathcal{W}_{M}^{s}$ at point $U_{M}$ that is tangent to the eigenvector $\left(1,-C_{M}, 0,0,0\right)$ of $A_{M}$. Hence, system (1.9) has a center-unstable manifold $\mathcal{W}_{m}^{c u}$ at point $U_{m}$ of dimension 4 that contains $\mathcal{W}_{m}^{u}$, and a center-stable manifold $\mathcal{W}_{M}^{c s}$ at point $U_{M}$ of dimension 4 that contains $\mathcal{W}_{M}^{s}$. Their intersection $\mathcal{M}:=\mathcal{W}_{m}^{c u} \cap \mathcal{W}_{M}^{c s}$ thus contains the orbit $\Gamma$. We have to show that $\mathcal{M}$ is a submanifold of dimension 3 of $\mathbb{R}^{5}$, which is equivalent to showing that the hypersurfaces $\mathcal{W}_{m}^{c u}$ and $\mathcal{W}_{M}^{c s}$ are transverse to each other along $\Gamma$. Therefore, we have to calculate their tangent spaces at each point of $\Gamma$. The hypersurface $\mathcal{W}_{m}^{c u}$ consists of the unstable curves emanating from the fixed points close to $U_{m}$, that is to say the points of $\mathcal{W}_{m}^{c}$. Hence, for all $\xi_{0} \in \mathbb{R}$, the tangent space $T_{\bar{U}\left(\xi_{0}\right)} \mathcal{W}_{m}^{c u}$ consists of the sum of $T_{\bar{U}\left(\xi_{0}\right)} \Gamma$ and the displaced space of $T_{U_{m}} \mathcal{W}_{m}^{c}$ along $\Gamma$ at point $\bar{U}\left(\xi_{0}\right)$. The vectors of the latter are the values at $\xi_{0}$ of solutions to the linearized system around $\Gamma$ :

$$
\alpha^{\prime}=\beta, \beta^{\prime}=\gamma-d_{v} p(V(\xi)) \alpha, \gamma^{\prime}=0, \delta^{\prime}=0, \eta^{\prime}=0,
$$

that have a limit at $-\infty$ belonging to $T_{U_{m}} \mathcal{W}_{m}^{c}$. In view of (1.8), we get for any solution to (1.10) having a limit at $-\infty$ :

$$
\alpha^{\prime} V^{\prime}-V^{\prime \prime} \alpha=\gamma\left(V-v_{m}\right) .
$$

Conversely, if $Q_{m}(\xi):=\int_{\xi_{0}}^{\xi}\left(V-v_{m}\right) /\left(V^{\prime}\right)^{2}$, then

$$
\lim _{\xi \rightarrow-\infty} V^{\prime}(\xi) Q_{m}(\xi)=-1 / C_{m}^{2} .
$$

So, for all $(\gamma, \delta, \eta) \in \mathbb{R}^{3}$ the function

$$
\xi \mapsto\left(\alpha(\xi)=\gamma V^{\prime}(\xi) Q_{m}(\xi), \beta(\xi)=\alpha^{\prime}(\xi), \gamma(\xi)=\gamma, \delta(\xi)=\delta, \eta(\xi)=\eta\right)
$$


is a solution to (1.10) that has the limit at $-\infty$ :

$$
\left(-\gamma / C_{m}^{2}, 0, \gamma, \delta, \eta\right) \in T_{U_{m}} \mathcal{W}_{m}^{c}
$$

Consequently, $T_{\bar{U}\left(\xi_{0}\right)} \mathcal{W}_{m}^{c u}$ is generated by

$$
\begin{gathered}
\left(V^{\prime}\left(\xi_{0}\right), V^{\prime \prime}\left(\xi_{0}\right), 0,0,0\right),\left(0,\left(V\left(\xi_{0}\right)-v_{m}\right) / V^{\prime}\left(\xi_{0}\right), 1,0,0\right), \\
(0,0,0,1,0) \text { and }(0,0,0,0,1) .
\end{gathered}
$$

A similar computation shows that $T_{\bar{U}\left(\xi_{0}\right)} \mathcal{W}_{M}^{c s}$ is generated by

$$
\begin{gathered}
\left(V^{\prime}\left(\xi_{0}\right), V^{\prime \prime}\left(\xi_{0}\right), 0,0,0\right),\left(0,\left(V\left(\xi_{0}\right)-v_{M}\right) / V^{\prime}\left(\xi_{0}\right), 1,0,0\right), \\
(0,0,0,1,0) \text { and }(0,0,0,0,1) .
\end{gathered}
$$

Since $v_{m} \neq v_{M}$, the hyperplanes $T_{\bar{U}\left(\xi_{0}\right)} \mathcal{W}_{m}^{c u}$ and $T_{\bar{U}\left(\xi_{0}\right)} \mathcal{W}_{M}^{c s}$ are different and thus transverse to each other. So the manifolds $\mathcal{W}_{m}^{c u}$ and $\mathcal{W}_{M}^{c s}$ are also transverse at point $\bar{U}\left(\xi_{0}\right)$. Their intersection $\mathcal{M}$ is a submanifold of dimension 3 , the tangent space of which is $T_{\bar{U}\left(\xi_{0}\right)} \mathcal{M}$, generated by

$$
\left(V^{\prime}\left(\xi_{0}\right), V^{\prime \prime}\left(\xi_{0}\right), 0,0,0\right),(0,0,0,1,0) \text { and }(0,0,0,0,1) .
$$

Furthermore, we see in the limit case that $T_{U_{m}} \mathcal{W}_{M}^{c s}$ is generated by

$$
\left(1, C_{m}, 0,0,0\right),(0,1,0,0,0),(0,0,0,1,0) \text { and }(0,0,0,0,1) \text {. }
$$

This proves that $\mathcal{W}_{M}^{c s}$ and $\mathcal{W}_{m}^{c}$ are transverse to each other at point $U_{m}$. Their intersection $\Sigma_{m}$ is also equal to $\mathcal{M} \cap \mathcal{W}_{m}^{c}$ and is a submanifold of dimension 2 of $\mathbb{R}^{5}$ at point $U_{m}$, the tangent plane of which is $T_{U_{m}} \Sigma_{m}$, generated by $(0,0,0,1,0)$ and $(0,0,0,0,1)$. Similarly, the intersection $\Sigma_{M}$ of $\mathcal{W}_{m}^{c u}$ and $\mathcal{W}_{M}^{c}$ is equal to $\mathcal{M} \cap \mathcal{W}_{m}^{c}$ and is a submanifold of dimension 2 of $\mathbb{R}^{5}$ at point $U_{M}$, the tangent plane of which is $T_{U_{M}} \Sigma_{M}=T_{U_{m}} \Sigma_{m}$.

Finally, each point $U_{l}$ of $\Sigma_{m}$ close to $U_{m}$ belongs to the stable manifold of a point $U_{r}$ of $\Sigma_{M}$, which belongs itself to the unstable manifold of $U_{l}$. This proves the existence of a heteroclinic orbit connecting these two fixed points. The surfaces $\Sigma_{m}$ and $\Sigma_{M}$ can be both parametrized by $(j, \nu)$ in such a way that these points read:

$$
\begin{gathered}
U_{l}=\left(v_{l}(j, \nu), 0, \pi(j, \nu)=p\left(v_{l}(j, \nu)\right)+j^{2} v_{l}(j, \nu), j, \nu\right), \\
U_{r}=\left(v_{r}(j, \nu), 0, \pi(j, \nu)=p\left(v_{r}(j, \nu)\right)+j^{2} v_{r}(j, \nu), j, \nu\right),
\end{gathered}
$$

with $d v_{l, r}(0,0)=(0,0)$.

We shall now compute the quadratic approximation of the surfaces $\Sigma_{m, M}$.

Remark 1.2. We know (see $\S 1.1$ ) that for all $\nu \geq 0$ we have

$$
v_{l}(0, \nu)=v_{m} \text { and } v_{r}(0, \nu)=v_{M} .
$$

In particular, we shall have $\partial_{\nu \nu}^{2} v_{l, r}(0,0)=0$.

Proposition 1.3. In the neighborhood of $(0,0)$ we have

$$
\begin{aligned}
& v_{l}(j, \nu)-v_{m}=\frac{1}{C_{m}^{2}}\left(v_{M}-v_{m}\right)\left(-\frac{1}{2} j^{2}+j \nu R\right)+\text { h.o.t, } \\
& v_{r}(j, \nu)-v_{M}=\frac{1}{C_{M}^{2}}\left(v_{M}-v_{m}\right)\left(+\frac{1}{2} j^{2}+j \nu R\right)+\text { h.o.t, }
\end{aligned}
$$

where $R:=\int_{-\infty}^{+\infty}\left(V^{\prime}\right)^{2} /\left(v_{M}-v_{m}\right)^{2}$. 
Proof. For $(j, \nu)$ close to $(0,0)$, we write the solution of (1.9) that takes the values $U_{l}(j, \nu)$ at $-\infty$ and $U_{r}(j, \nu)$ at $+\infty$ as

$$
U=\bar{U}+U_{1}+U_{2}+o\left(U_{2}\right)
$$

where $U_{1}$ is a solution to the first order approximate system (1.10). We recall from Lemma 1 that $U_{1}=\left(\alpha_{1}, \beta_{1}, \gamma_{1}, \delta_{1}, \eta_{1}\right)$ has to satisfy

$$
\alpha_{1}^{\prime} V^{\prime}-V^{\prime \prime} \alpha_{1}=\gamma_{1}\left(V-v_{m}\right)=\gamma_{1}\left(V-v_{M}\right) \text {. }
$$

This implies $\gamma_{1}=0$ and the existence of $a_{1} \in \mathbb{R}$ such that $\alpha_{1}=a_{1} V^{\prime}$. Furthermore, the system satisfied by $U_{2}=\left(\alpha_{2}, \beta_{2}, \gamma_{2}, \delta_{2}, \eta_{2}\right)$ is

$$
\begin{gathered}
\alpha_{2}^{\prime}=\beta_{2}, \gamma_{2}^{\prime}=0, \delta_{2}^{\prime}=0, \eta_{2}^{\prime}=0 \\
\beta_{2}^{\prime}=\gamma_{2}-d_{v} p(V(\xi)) \alpha_{2}-d_{v v}^{2} p(V(\xi)) \alpha_{1}^{2} / 2+\delta_{1} \eta_{1} V^{\prime}-\delta_{1}^{2} V .
\end{gathered}
$$

Some elementary computations then show that:

$$
\left(\beta_{2} V^{\prime}-(\bar{p}-p(V)) \alpha_{2}-\gamma_{2} V+\frac{1}{2} \delta_{1}^{2} V^{2}\right)^{\prime}=\left(\delta_{1} \eta_{1}+\frac{1}{2} a_{1}^{2} E(V)^{\prime}\right)\left(V^{\prime}\right)^{2}
$$

where $d_{v} E=-p$. Now we note from (1.8) that

$$
\int_{-\infty}^{+\infty} E(V)^{\prime}\left(V^{\prime}\right)^{2}=0
$$

The integration of (1.14) thus implies

$$
-\gamma_{2}\left(v_{M}-v_{m}\right)+\frac{1}{2} \delta_{1}^{2}\left(v_{M}^{2}-v_{m}^{2}\right)=\delta_{1} \eta_{1} \int_{-\infty}^{+\infty}\left(V^{\prime}\right)^{2}
$$

Since the third component of $U$ is $\pi(j, \nu)=\bar{p}+\gamma_{2}+o\left(U_{2}\right)$ while $j=\delta_{1}+\delta_{2}+$ $o\left(U_{2}\right)$ and $\nu=\eta_{1}+\eta_{2}+o\left(U_{2}\right)$, we deduce from (1.15) that for $(j, \nu)$ close to $(0,0)$ :

$$
\pi(j, \nu)-\bar{p} \sim \frac{1}{2} j^{2}\left(v_{M}+v_{m}\right)-j \nu \int_{-\infty}^{+\infty}\left(V^{\prime}\right)^{2} /\left(v_{M}-v_{m}\right) .
$$

The aimed result (1.12) is then obtained by expanding up to second order the relations

$$
\pi(j, \nu)=p\left(v_{l}(j, \nu)\right)+j^{2} v_{l}(j, \nu)=p\left(v_{r}(j, \nu)\right)+j^{2} v_{r}(j, \nu) .
$$

Remark 1.4. The expansions (1.12) are in accordance with the behavior pointed out in paragraph 1.1. In particular, for $\nu=0$ we have

$$
\frac{d v_{r}}{d v_{l}}\left(v_{m}\right)=-\frac{C_{m}^{2}}{C_{M}^{2}}<0
$$

whereas for $\nu=$ cte $>0$ we have

$$
\frac{d v_{r}}{d v_{l}}\left(v_{m}\right)=\frac{C_{m}^{2}}{C_{M}^{2}}>0
$$




\section{Nonuniqueness of PHASE transitions}

In view of (1.11) the functions $\left(v_{l, r}-v_{m, M}\right) / j$ are smooth, as well as $\partial_{j} v_{l, r}$. By applying the Implicit Function Theorem to these functions, we easily deduce from Proposition 1.2:

Proposition 2.1. There exist four smooth functions of $\nu$ in the neighborhood of 0 such that

$$
\begin{gathered}
j_{M}(\nu)<j_{\operatorname{Min}}(\nu)<0<j_{\max }(\nu)<j_{m}(\nu), \\
j_{M}(\nu) \sim-2 R \nu, j_{\operatorname{Min}}(\nu) \sim-R \nu, j_{\max }(\nu) \sim R \nu, j_{m}(\nu) \sim 2 R \nu,
\end{gathered}
$$

and

$$
v_{l}\left(j_{m}(\nu), \nu\right)=v_{m}
$$

$\partial_{j} v_{l}>0 \quad$ if $j_{M}(\nu) \leq j<j_{\max }(\nu)$ and $\partial_{j} v_{l}<0$ if $j_{\max }(\nu)<j \leq j_{m}(\nu)$,

$$
v_{r}\left(j_{M}(\nu), \nu\right)=v_{M}
$$

$\partial_{j} v_{r}<0 \quad$ if $j_{M}(\nu) \leq j<j_{M i n}(\nu)$ and $\partial_{j} v_{r}>0$ if $j_{M i n}(\nu)<j \leq j_{m}(\nu)$.

(The reader may refer to Figure 2.) We are now in a position to state the

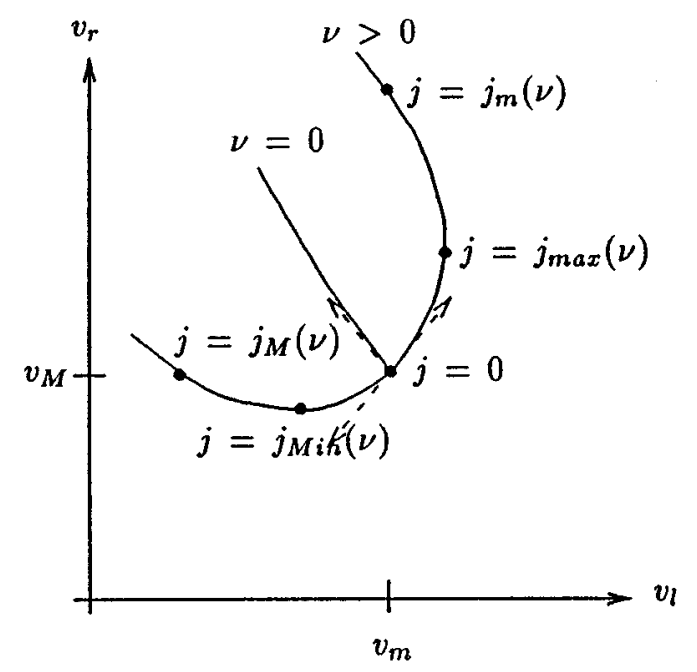

Figure 2. Admissible phase transitions close to $\left(v_{m}, v_{M}\right)$

Theorem 2.2. Let $p: v \in] b,+\infty\left[\mapsto p(v)>0\right.$ be of class $\mathcal{C}^{2}$ such that

$$
\begin{gathered}
\left.d_{v} p<0 \quad \text { if } v \in\right] b, v_{\star}[\cup] v^{\star},+\infty\left[, d_{v} p>0 \text { if } v \in\right] v_{\star}, v^{\star}[, \\
\lim _{v \rightarrow b} p(v)=+\infty, \lim _{v \rightarrow+\infty} p(v)=0 .
\end{gathered}
$$

Let $v_{m}$ and $v_{M}$ be defined by (1.1). Then there exists $\nu_{0}>0$ such that for all $\nu \in] 0, \nu_{0}\left[\right.$ there are four values $v_{\min }, v_{\max }, v_{\text {Min }}$ and $v_{\text {Max }}$ - depending on $\nu-$ satisfying

$$
v_{\min }<v_{m}<v_{\max }<v_{\star}, v^{\star}<v_{\text {Min }}<v_{M}<v_{\text {Max }}
$$


such that

- for all $v_{l} \in\left[v_{m}, v_{\max }\left[\right.\right.$ there are two different pairs $\left(v_{r}, j\right) \in\left[v_{M}, v_{\text {Max }}\right] \times$ $\mathbb{R}^{+}$such that the problem (1.5)-(1.6) has a solution,

- for all $\left.\left.v_{r} \in\right] v_{M i n}, v_{M}\right]$ there are two different pairs $\left(v_{l}, j\right) \in\left[v_{\min }, v_{m}\right] \times$ $\mathbb{R}^{-}$such that the problem (1.5)-(1.6) has a solution.

Proof. This is a direct consequence of Lemma 1.1 and Propositions $1.2-2.1$, with

$$
\begin{array}{ll}
v_{\max }(\nu):=v_{l}\left(j_{\max }(\nu), \nu\right), & v_{\operatorname{Max}}(\nu):=v_{r}\left(j_{m}(\nu), \nu\right), \\
v_{\text {Min }}(\nu):=v_{r}\left(j_{\operatorname{Min}}(\nu), \nu\right), & v_{\min }(\nu):=v_{l}\left(j_{M}(\nu), \nu\right) .
\end{array}
$$

Remark 2.3. From (2.1) and (1.12), we have the estimates:

$$
\begin{aligned}
& v_{\max }(\nu)-v_{m} \sim+\frac{1}{2 C_{m}^{2}}\left(v_{M}-v_{m}\right) R^{2} \nu^{2}, \\
& v_{M a x}(\nu)-v_{M} \sim+\frac{4}{C_{M}^{2}}\left(v_{M}-v_{m}\right) R^{2} \nu^{2}, \\
& v_{M i n}(\nu)-v_{M} \sim-\frac{1}{2 C_{M}^{2}}\left(v_{M}-v_{m}\right) R^{2} \nu^{2}, \\
& v_{\min }(\nu)-v_{m} \sim-\frac{4}{C_{m}^{2}}\left(v_{M}-v_{m}\right) R^{2} \nu^{2}
\end{aligned}
$$

\section{REFERENCES}

1. S. Benzoni-Gavage, Stability of multidimensional phase transitions. Nonlinear Analysis T.M.A., 31, no. 1/2 (1998), pp. 243-263. CMP 98:06

2. H. Fan \& M. Slemrod, The Riemann problem for systems of conservation laws of mixed type. IMA Vol. Math. Appl., 52 (1993), pp. 61-91. MR 94h:35152

3. H. Freistühler, The Persistence of Ideal Shock Waves. Appl. Math. Lett., 7 no 6 (1994), pp. 7-11. MR 96c:35114

4. M. Grinfeld, Dynamic phase transitions: Existence of "cavitation waves". Proc. Roy. Soc. Edinburgh Sect. A, 107 (1987), pp. 153-163. MR 88k:35123

5. D. Serre, Systems of conservation laws. Cambridge University Press, to appear.

6. M. Slemrod, Admissibility criteria for propagating phase boundaries in a van der Waals fluid. Arch. Rational Mech. Anal. 81 (1983), pp. 301-315. MR 84a:76030

7. M. Shearer, Admissibility criteria for shock wave solutions of a system of conservation laws of mixed type. Proc. Roy. Soc. Edinburgh 93 A (1983), pp. 233-244. MR 84c:35075

8. M. Shearer, Nonuniqueness of admissible solutions of Riemann initial value problems for a system of conservation laws of mixed type. Arch. Rational Mech. Anal. 93 (1986), pp. 45-59. MR 87h:35207

CNRS-ENS Lyon, UMR 128, 46, allée D’Italie, F-69364 Lyon Cedex 07, France

E-mail address: benzoni@umpa.ens-lyon.fr 